\title{
Practical Cryptanalysis of the Identification Scheme Based on the Isomorphism of Polynomial with One Secret Problem
}

\author{
Charles Bouillaguet $^{1}$, Jean-Charles Faugère ${ }^{2,3}$, Pierre-Alain Fouque $^{1}$, \\ and Ludovic Perret $^{3,2}$ \\ ${ }^{1}$ Ecole Normale Supérieure, Paris, France \\ \{charles.bouillaguet, pierre-alain. fouque\} @ens.fr \\ 2 INRIA, Paris-Rocquencourt Center, SALSA Project \\ UPMC Univ. Paris 06, UMR 7606, LIP6, F-75005, Paris, France \\ 3 CNRS, UMR 7606, LIP6, F-75005, Paris, France \\ jean-charles.faugere@inria.fr, ludovic.perret@lip6.fr
}

\begin{abstract}
This paper presents a practical cryptanalysis of the Identification Scheme proposed by Patarin at Crypto 1996. This scheme relies on the hardness of the Isomorphism of Polynomial with One Secret (IP1S), and enjoys shorter key than many other schemes based on the hardness of a combinatorial problem (as opposed to number-theoretic problems). Patarin proposed concrete parameters that have not been broken faster than exhaustive search so far. On the theoretical side, IP1S has been shown to be harder than Graph Isomorphism, which makes it an interesting target. We present two new deterministic algorithms to attack the IP1S problem, and we rigorously analyze their complexity and success probability. We show that they can solve a (big) constant fraction of all the instances of degree two in polynomial time. We verified that our algorithms are very efficient in practice. All the parameters with degree two proposed by Patarin are now broken in a few seconds. The parameters with degree three can be broken in less than a CPU-month. The identification scheme is thus quite badly broken.
\end{abstract}

\section{Introduction}

Multivariate cryptography is concerned with the use of multivariate polynomials over finite fields to design cryptographic schemes. The use of polynomial systems in cryptography dates back to the mid eighties with the design of $\mathrm{C}^{*}$ [33], and many others proposals appeared afterwards $[37|38| 39|28| 47]$. The security of multivariate schemes is in general related to the difficulty of solving random or structured systems of multivariate polynomial equations. This problem has been proved to be NP-complete [22], and it is conjectured [2] that systems of random polynomials are hard to solve in practice. As usual when a trapdoor must be embedded in a hard problem, easy instances are transformed into random-looking instances using secret transformations. In multivariate cryptography, it is common to map an easily-invertible collection of polynomials a into an apparently random one $\mathbf{b}$. It is then assumed that, being supposedly indistinguishable from random, $\mathbf{b}$ should be hard to solve. The structure-hiding transformation is

D. Catalano et al. (Eds.): PKC 2011, LNCS 6571, pp. 473 493, 2011.

(C) International Association for Cryptologic Research 2011 
very often the composition with linear (or affine) invertible mappings $S$ and $T$, namely $\mathbf{b}=T \circ \mathbf{a} \circ S$. The matrices $S$ and $T$ are generally part of the secret-key.

The Isomorphism of Polynomials (IP) is the problem of recovering the secret transformations $S$ and $T$ given a and $\mathbf{b}$. It is a fundamental problem of multivariate cryptography, since its hardness implies the difficulty of the key-recovery for various multivariate cryptosystems. Notorious examples include $C^{*}$ [33], the traitor tracing scheme proposed by Billet and Gilbert [8], the SFLASH signature scheme [38], the $\ell$-IC signature scheme [12], the square-vinegar signature scheme [1] and the Square encryption scheme [11] 1 . All these schemes have been broken, because the structure of the central map was not hidden well enough. The corresponding IP problem was then not random, but structured. However, when no apparent structure exists in both a and $\mathbf{b}$, then the IP problem is fairly difficult. This motivated Patarin to introduce it as an intractable assumption by itself in [35]. So far only exponential algorithms [40,17] are known to attack the general IP problem.

An important special case of IP is the IP problem with one secret (IP1S for short), where $T$ is the identity matrix. Patarin suggested in 1996 [36] to construct a zeroknowledge identification scheme relying on the hardness of IP1S, inspired by the ZeroKnowledge proof system for Graph Isomorphism of [25]. The proposed parameters lead to relatively small key sizes (for instance to secret and public keys of 256 bits each and no additional information), as the complexity of the problem was believed to be exponential. The proposed parameters have not been broken so far, and no technique better than exhaustive search is known to attack the scheme. The IP1S problem is also interesting from a complexity-theoretic point of view. It has been proved in [40] that IP1S is Graph Isomorphism-hard (GI-hard for short). This leads Patarin et al. to claim that IP1S is unlikely to be solvable in polynomial time, because no polynomial algorithm is known for GI in spite of more than forty years of research. On the other hand, GI is not known to be NP-complete. Generating hard instances GI is pretty non-trivial, and there are powerful heuristics as well as expected linear time algorithms for random graphs [19]. This compromises the use of GI as an identification mechanism, and was part of the motivation for introducing IP1S as an alternative. Moreover, when used in this context, instances of the IP problem are random, which presumably avoids all the attacks on the cryptographic schemes mentioned above.

Previous and Related Work. The identification scheme based on IP1S is not based on number-theoretic assumptions, unlike for instance the well-known Fiat-Shamir protocol [18]. Many other identification schemes are not based on number theoretic assumptions [42 43 44 4531]. However, the IP1S-based identification scheme enjoys shorter keys than most others.

To our knowledge, the first algorithm dedicated to IP1S can be found in Geiselmann et al. [23]. The authors of [23] remarked that each row of a matrix solution of IP1S verifies an algebraic system of equations. They then used an exhaustive search to find the solutions of such system. Soon after, this technique has been improved by Levy-dit-Vehel and Perret [13] who replaced this exhaustive search by a Gröbner basis

\footnotetext{
${ }^{1}$ In the description of some of these schemes, the easily-invertible central map contains parameters that are part of the secret-key. However, in this case there exists an equivalent secret key where these parameters have a fixed value. This is notoriously the case of $\mathrm{C}^{*}$.
} 
computation. This still yields exponential algorithms, and the improvement induced by this replacement is is as significant as the gain obtained when comparing Gröbner basis and exhaustive search for solving random algebraic systems. It is negligible over small field (i.e., typically, $\mathbb{F}_{2}$ ), but significant for instances of IP1S over large fields. However, the complexity of those algorithms remains exponential by nature.

Finally, Perret [41] shows that the affine and linear variants of IP1S are equivalent, i.e., one can without loss of generality restrict our attention to the case where $S$ is linear (as opposed to affine). In addition, a new approach for solving IP1S using the Jacobian matrix was proposed. The algorithm is polynomial when the number $u$ of polynomials in $\mathbf{a}$ and $\mathbf{b}$ is equal to the number of variables $n$. However, when $u<n$, the complexity of this approach is not well understood. Moreover, when the number of polynomials is very small, for instance $u=2$, this algorithm is totally inefficient.

The main application of IP1S is the identification scheme proposed in [40]. The public key being composed of two sets of $u$ polynomials, it is interesting to keep the number of polynomials as small as possible (1 or 2 ). For such parameters, the authentication mechanism based on IP1S looks appealing in terms of key size. Additionally, it does not require hash functions or commitments.

All in all, the existing literature on the IP and IP1S problem can be split in two categories: heuristic algorithms with (more or less vaguely) "known" complexity and unknown success probability [40], and rigorous algorithms that always succeeds but with unknown complexity [1741,1323]. This situation makes it very difficult, if not plainly impossible to compare these algorithms based on their theoretical features. The class of instances that can be solved by a given algorithm of the first type is in general not known. Conversely, the class of instances over which an algorithm of the second type terminates quickly are often not known as well. This lead the authors of IP/IP1S algorithms to measure the efficiency of their techniques in practice, or even not to measure it at all. Several sets of concrete parameters for IP and IP1S were proposed by Patarin in [36], and can be used to measure the progress accomplished since their introduction. The techniques presented in this paper allow to break all these challenges in practice.

Techniques. The algorithms presented here are deterministic, and rely on the two weapons that have dealt a severe blow to multivariate cryptography: linear algebra and Gröbner bases. Our ideas borrow to the recent differential cryptanalysis of multivariate schemes. While the algorithms are not very complicated, analyzing their running time is fairly non-trivial, and requires the invocation of not-so-well-known results about linear algebra (such as the dimension of the commutant of a matrix, or the properties of the product of two skew-symmetric matrices), as well as known results about random matrices, most notably the distribution of the rank and the probability of being cyclic. The two most delicate steps of the analysis involve lower-bounding the dimension of the kernel of a homogeneous system of matrix equations, and upper-bounding the degree of polynomials manipulated by a Gröbner-basis algorithm.

Our Results. We present two new "rigorous" and deterministic algorithms. On the practical side, these algorithms are efficient: random quadratic IP1S instances and random cubic inhomogeneous IP1S instances can be broken in time $\mathcal{O}\left(n^{6}\right)$ for any size of the parameters. In particular, all the quadratic IP1S challenges proposed by Patarin 
are now broken in a few seconds. The biggest homogeneous cubic IP1S challenge can be broken in less than 1 CPU-month. The IP1S identification scheme is thus broken beyond repair in the quadratic case. In the case of cubic IP1S, our attack runs in time $\mathcal{O}\left(n^{6} \cdot q^{n}\right)$, and the security parameter have to be seriously reconsidered, which makes the scheme much less attractive, since the key size is cubic in $n$.

A rigorous analysis of our algorithms is both necessary and tricky. When generating linear equations, special care has to be taken to count how many of them are independent. The recent history of algebraic cryptanalysis taught us that failure to do so may have drastic consequences. Additionally, the complexity of Gröbner bases computation, even though a bit more well-understood now in the generic case, is still often a delicate matter for structured systems.

A unique and distinctive feature of our algorithms compared to the previous state of affairs, and one of our main theoretical contribution, is that we characterize the class of instances that can be solved by our techniques in polynomial time. We show, for instance, that a (big) constant fraction of all quadratic IP1S instances can be solved in polynomial time.

This break however has little consequences in the multivariate cryptology ecosystem, except that it brings the IP1S-based identification scheme down. The security of UOV [27] in particular is not related to the hardness of IP1S, because in UOV the vector of polynomial composed with a linear change of variable (the " $a$ " part) is kept secret.

Organisation of the paper. In section 2, we recall some useful facts about the IP1S problem. Then, in section 3 we introduce the identification scheme based on the hardness of IP1S and compare it to other non-number theoretic based ID schemes. We then introduce our algorithms to break IP1S in the quadratic case in section 4 , and in the cubic case in section 5

\section{The IP1S Problem}

We recall the definition of the IP1S problem. Given two families of $u$ polynomials a and $\mathbf{b}$ in $\mathbb{F}_{q}\left[x_{1}, \ldots, x_{n}\right]$ the task is to find an invertible matrix $S \in \mathrm{GL}_{n}\left(\mathbb{F}_{q}\right)$ and a vector $c \in\left(\mathbb{F}_{q}\right)^{n}$ such that:

$$
\mathbf{b}(\mathbf{x})=\mathbf{a}(S \cdot \mathbf{x}+c) .
$$

We will denote by $f^{(k)}$ the homogeneous component of degree $k$ of $f$, and by extension $\mathbf{a}^{(k)}$ denotes the vector of polynomials obtained by taking the homogeneous components of degree $k$ of all the coordinates of $\mathbf{a}$. We define the derivative of a in $c$ to be the function $\frac{\partial \mathbf{a}}{\partial c}: \mathbf{x} \mapsto \mathbf{a}(\mathbf{x}+c)-\mathbf{a}(\mathbf{x})$. The following lemma, which is very similar to [17, lemma 4] is very useful.

Lemma 1. i) For all $k \geq 1$, we have:

$$
\mathbf{b}^{(k)}=\left(\mathbf{a}+\frac{\partial \mathbf{a}}{\partial c}\right)^{(k)} \circ S .
$$


ii) If $d$ is the degree of $\mathbf{a}$ and $\mathbf{b}$, then $\mathbf{b}^{(d)}=\mathbf{a}^{(d)} \circ S$.

iii) $S$ transforms the set of common zeroes of $\mathbf{a}^{(d)}$ into the set of common zeroes of $\mathbf{b}^{(d)}$.

Proof. It follows from the definition of the derivative that:

$$
\mathbf{b}=\left(\mathbf{a}+\frac{\partial \mathbf{a}}{\partial c}\right) \circ S
$$

This equality also holds if only the degree- $k$ homogeneous component is considered. The point is that since $S$ is linear (and thus not "degree-changing"), if $P$ is a multivariate polynomial we have:

$$
(P \circ S)^{(k)}=P^{(k)} \circ S
$$

This establishes the first statement of the lemma. The second statement follows from the fact that if $\mathbf{a}$ is of degree $d$, then the function $\frac{\partial \mathbf{a}}{\partial c}$ is of degree $d-1$. Thus the homogeneous component of degree $d$ of $\frac{\partial \mathbf{a}}{\partial c}$ is identically zero. The third statements is a direct consequences of the second one.

A useful consequence of lemma 1 is that without loss of generality we may assume $c$ to be the null vector. A consequence of point $i i$ ) is that from any instance of the problem we can deduce a linear homogeneous instance by considering only the homogeneous component of highest degree. If this instance can be solved, and $S$ can be retrieved, then recovering $c$ is not difficult, using a slight generalization of the idea shown in [24]. If $S$ is known, then $\frac{\partial \mathbf{a}}{\partial c}$ can be explicitly computed, and $c$ can usually be deduced therefrom. More specifically, focusing on the homogeneous component of degree one yields a system of $u \cdot n$ linear equations in $n$ variables that admits $c$ as a solution. In most cases, it will in fact admit only $c$ as a solution, which enables recovering $c$.

It was pointed out in [40] that if there is only one quadratic polynomial, then the problem is easily solved in polynomial time. This follows from the fact that quadratic forms admit a canonical representation (see for instance [30]). The change of coordinate can then be easily computed. We will therefore focus on the case of $u \geq 2$ when the polynomials are quadratic.

For various reasons, the IP1S problem becomes easier when $u$ is close to $n$, and harder when $u$ is small. For instance, the algorithm given in [41] deals with the case $u=n$ in polynomial time, but cannot tackle the case where $u=2$ and $n$ is big, which prevented it from breaking the parameters proposed by Patarin. Additionally, small values of $u$ leads to smaller public keys. Therefore, we will restrict our attention to the case where $u=2$ when the polynomials are quadratic, and where $u=1$ when they are cubic. These are the most cryptographically relevant cases, and the most challenging. We will also consider the case where $\mathbb{F}_{q}$ is a field of characteristic two. It can be shown that this makes the problem a bit harder, but again this is the most cryptographically relevant case. The quadratic and cubic IP1S problems are very different and lead to specific approaches, therefore we will discuss them separately.

${ }^{2}$ This was already observed in [41]. 


\section{Patarin's IP1S-Based Identification Scheme}

Zero-Knowledge proofs were introduced in 1985 by Goldwasser, Micali and Rackoff in [26]. Soon afterwards, Fiat and Shamir [18] used the hardness of quadratic residuosity to build an efficient identification scheme. Many other identification schemes appeard afterwards, all relying on the hardness of number-theoretic assumptions. Some cryptographers took a different line of research, and tried to design identification scheme from different computational assumptions, not relying on number theory, but instead on the NP-hardness of some specific combinatorial problems.

One of the very-first combinatorial identification scheme was proposed by Shamir [43], and relied on the hardness of the Permuted Kernel Problem (PKP). Later on, Stern proposed in [44] a scheme based on the intractability of Syndrome Decoding (SD), and in [45] a scheme based on the intractability of Constrained Linear Equations (CLE). Finally, Pointcheval [42] proposed a scheme related to the hardness of the Perceptron Problem, originating from the area of learning theory. All these problems are NP-complete (as opposed to IP1S). The designers proposed practical parameters, aiming for a security level of $2^{64}$ or more, which are summarized in table 1 In all these schemes, it is required that all users share a public common set of information, a "common setting", usually describing the instance of the hard problem. For instance, in number-theoretic problems, the description of the curve, or of the group over which a discrete logarithm problem is considered is a common public information. While this information is not a "key" stricto sensu, it must nevertheless be stored by the prover and by the verifier, leading to higher memory requirements. However, in some case it can be chosen randomly, or generated online from a small seed using a PRNG.

Table 1. Key sizes in bits corresponding to practical parameters proposed in $[42|43| 44|45| 36]$ in order to obtain a security level of roughly $2^{64}$

\begin{tabular}{|c|c|c|c|}
\hline Scheme & Common Setting & Public Key & Secret Key \\
\hline \hline \multirow{2}{*}{ PKP } & 2048 & 256 & 374 \\
& 7992 & 512 & 808 \\
\hline \multirow{2}{*}{ SD } & 131072 & 256 & 512 \\
& 524288 & 512 & 1024 \\
\hline \multirow{2}{*}{ CLE } & 3600 & 80 & 80 \\
& 3600 & 96 & 96 \\
\hline Perceptron & 10807 & 144 & 117 \\
\hline \hline IP1S & 0 & 256 & 272 \\
\hline
\end{tabular}

On the contrary, the IP1S-based identification scheme proposed by Patarin in [35[36] does not need the prover and the verifier to share additional information (except maybe the description of the finite field, which is very small). It works very similarly to the original identification scheme based on a zero-knowledge proof system for GraphIsomorphism (GI) by Goldreich, Micali and Wigderson [25]. One of the reasons for replacing GI by IP1S is the existence of efficient heuristic algorithms for GI, capable of solving efficiently random instances. The generation of hard instances of GI is a delicate matter [19]. Replacing the GI problem by IP1S yields shorted key, and random 
Table 2. Concrete parameters for IP1S. Patarin proposed challenges A,B,C and D in [36]. We introduce challenge $\mathrm{E}$.

\begin{tabular}{|c||c|c|c|c|c|c|}
\hline Challenge & $n$ & $q$ & Degree & Polynomial(s) & Public Key & Private Key \\
\hline \hline A & 16 & 2 & 2 & 2 & 272 bits & 256 bits \\
\hline B & 16 & 2 & 3 & 1 & 816 bits & 256 bits \\
\hline C & 6 & 16 & 2 & 2 & 168 bits & 144 bits \\
\hline D & 6 & 16 & 3 & 1 & 224 bits & 144 bits \\
\hline E & 32 & 2 & 2 & 2 & 1056 bits & 1024 bits \\
\hline
\end{tabular}

instances of IP1S were a priori secure. Patarin proposed concrete parameters, which are shown in table. 2. The PKP and SD schemes lead to bigger keys than IP1S, while the Perceptron scheme leads to comparable key-sizes, and CLE yields smaller keys than IP1S, if we neglect the additional memory requirement imposed by the common string shared between all the participants.

Additionnaly, the IP1S-based identification scheme does not makes use of either hash functions or commitment schemes. This is in strong contrast with all the other proposals.

The IP1S challenges described in table 2 cannot be attacked using the existing techniques [17/23 41]. As such, the best attack remains exhaustively searching for the secret key. As a final note, let us mention that Lyubashevsky recently proposed in [31] to build an identification scheme using the hardness of lattice problems, but did not propose concrete parameters.

\section{Cryptanalysis of Quadratic IP1S}

The main observation underlying our quadratic IP1S algorithm is that by differentiating equation (1), it is possible to collect linear equations between the coefficients of $S$ and those of $S^{-1}$.

We denote by $\mathrm{D} f:\left(\mathbb{F}_{q}\right)^{n} \times\left(\mathbb{F}_{q}\right)^{n} \rightarrow \mathbb{F}_{q}{ }^{u}$ the differential of a function $f: \mathbb{F}_{q}^{n} \rightarrow \mathbb{F}_{q}^{u}$. $\mathrm{D} f$ is defined by:

$$
\mathrm{D} f(\mathbf{x}, \mathbf{y})=f(\mathbf{x}+\mathbf{y})-f(\mathbf{x})-f(\mathbf{y})+f(0)
$$

It is easy to see that $\operatorname{D} f(\mathbf{x}, \mathbf{y})=\operatorname{D} f(\mathbf{y}, \mathbf{x})$. If $f$ is a polynomial of total degree $d$, then $\mathrm{D} f$ is a polynomial of total degree $d$, but of degree $d-1$ in $\mathbf{x}$ and $\mathbf{y}$. Thus, when $f$ is quadratic, then $\mathrm{D} f$ is a symmetric bilinear mapping.

Going back to the quadratic IP1S problem, for all vectors $\mathbf{x}, \mathbf{y} \in\left(\mathbb{F}_{q}\right)^{n}$, we have:

$$
\forall \mathbf{x}, \mathbf{y} \in\left(\mathbb{F}_{q}\right)^{n}, \quad \operatorname{Db}(\mathbf{x}, \mathbf{y})=\operatorname{Da}(S \cdot \mathbf{x}, S \cdot \mathbf{y}) .
$$

Using the change of variable $\mathbf{y}^{\prime}=S \cdot \mathbf{y}$, this equation becomes:

$$
\forall \mathbf{x}, \mathbf{y}^{\prime} \in\left(\mathbb{F}_{q}\right)^{n}, \quad \operatorname{Db}\left(\mathbf{x}, S^{-1} \cdot \mathbf{y}^{\prime}\right)=\operatorname{Da}\left(S \cdot \mathbf{x}, \mathbf{y}^{\prime}\right) .
$$

Since $\mathbf{a}$ and $\mathbf{b}$ are of total degree 2, then $\mathrm{Da}$ and $\mathrm{Db}$ are bilinear (symmetric) mappings. In this case, since equation (2) is valid for all $\mathbf{x}$ and $\mathbf{y}$, then in particular it is valid on 
a basis of $\left(\mathbb{F}_{q}\right)^{n} \times\left(\mathbb{F}_{q}\right)^{n}$, and substituting fixed basis vectors for $\mathbf{x}$ and $\mathbf{y}$ yields linear equations between the coefficients of $S$ and those of $S^{-1}$.

This idea for obtaining linear equations can also be described relatively simply using the usual theory of quadratic forms. If $\mathbb{F}_{q}$ is a field of even (resp. odd) characteristic, then the set of homogeneous quadratic polynomials in $n$ variables over $\mathbb{F}_{q}$ is in oneto-one correspondance with the set of symmetric matrices with zero diagonal (resp. of symmetric matrices). Let $\mathcal{P}\left(\mathbf{a}_{k}\right)$ denote the matrix of the symmetric bilinear form associated with $\mathbf{a}_{k}$ (it is related to the polar form of $\mathbf{a}_{k}$ in odd characteristic). Recall that the coefficient of index $(i, j)$ of $\mathcal{P}\left(\mathbf{a}_{k}\right)$ is $\operatorname{Da} \mathbf{a}_{k}\left(e_{i}, e_{j}\right)$, where $\left(e_{i}\right)_{1 \leq i \leq n}$ is a basis of $\left(\mathbb{F}_{q}\right)^{n}$. We then have:

$$
\mathcal{S}: \quad\left\{\begin{array}{c}
S^{-1} \cdot \mathcal{P}\left(\mathbf{b}_{1}\right)=\mathcal{P}\left(\mathbf{a}_{1}\right) \cdot{ }^{t} S \\
\vdots \\
S^{-1} \cdot \mathcal{P}\left(\mathbf{b}_{u}\right)=\mathcal{P}\left(\mathbf{a}_{u}\right) \cdot{ }^{t} S
\end{array}\right.
$$

Each one of these $u$ matrix equations yields $n^{2}$ linear homogeneous equations between the $2 n^{2}$ coefficients of $S$ and those of $S^{-1}$. These last $u \cdot n^{2}$ homogeneous linear equations cannot be linearly independent as they admit a non-trivial solution $\left(S^{-1}, S\right)$. The kernel of $\mathcal{S}$ is thus non-trivial, and our hope would be that it describes only one solution. When $u$ is strictly greater than two, we then have much more linear equations than unknowns, and we empirically find only one solution (when the polynomials are randomly chosen). When $u=2$, which is again the most relevant case, the situation is unfortunately not as nice; Theorem 1 below shows that the kernel of $\mathcal{S}$ is of dimension higher that $2 n$ in characteristic two (at least $n$ in odd characteristic). This means that solving the linear equations cannot by itself reveal the solution of the IP1S problem, because $\mathcal{S}$ admits at least $q^{n}$ solutions, out of which only very few are actual solutions of the IP1S instance 3 . However, the linear equations collected this way can be used to simplify the resolution of the IP1S problem.

When looking at one coordinate of (1), we have an equality between two multivariate polynomials that holds for any value of the variables. Therefore the coefficients of the two polynomials can be identified (this is essentially the algorithm presented in [17]). This yields a system $\mathcal{S}_{\text {quad }}$ of $u \cdot n^{2} / 2$ quadratic equations in $n^{2}$ unknown over $\mathbb{F}_{q}$. With $u=2$, this precisely gives $n^{2}$ equations in $n^{2}$ unknown, which cannot be solved by any existing techniques faster than exhaustive search.

However, we now know that $\left(S, S^{-1}\right)$ lives in the kernel of $\mathcal{S}$, and therefore $S$ can be written as the sum of $k=\operatorname{dim} \operatorname{ker} \mathcal{S}$ matrices that can be easily computed using standard linear algebra. Identifying coefficients in (1) then yields a system $\mathcal{S}_{\text {quad }}$ of $u \cdot n^{2} / 2$ quadratic equations in $k$ unknown. Our hope is that $k$ is small enough for the system to be very overdetermined, so that computing a Gröbner basis of $\mathcal{S}_{\text {quad }}$ is polynomial in theory, and feasible in practice.

The analysis of the attack then proceeds in two steps:

1. Estimate the rank of $\mathcal{S}$ (i.e., the value of $k$ ).

2. Estimate the complexity of the Gröbner basis computation.

\footnotetext{
${ }^{3}$ We note that this contradicts the hope expressed in section 9 of [40].
} 

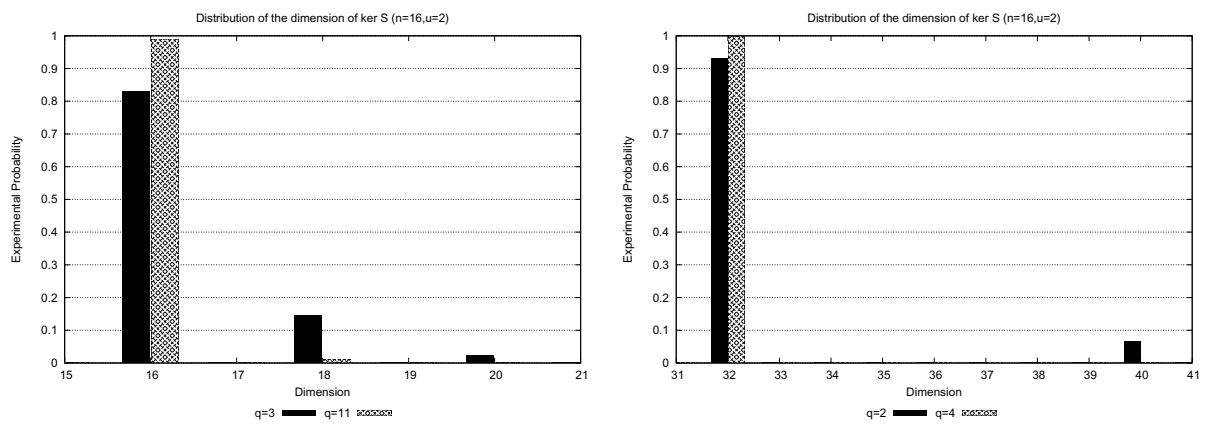

Fig. 1. Experimental distribution of $\operatorname{dim} \operatorname{ker} \mathcal{S}$

For the sake of simplicity, we will analyze the attack algorithm under some assumptions on the input system. For instance, we will assume that that $n$ is even, and that one of the two quadratic forms we are dealing with is non-degenerate. We will then argue that a random instance satisfies this assumption with high probability, but we are well aware that some structured instance may not. This is in fact quite logical, because a worst-case polynomial algorithm for IP1S would imply a worst-case polynomial for Graph-Isomorphism (a fact that would be quite surprising). The situation of the IP1S problem is in this respect quite similar to that of GI: heuristics are capable of dealing efficiently with the random case, while some very special instances make them fail (interestingly, hard instances for GI are transformed into hard instances for IP1S through the reduction). Lastly, we mention that our algorithm does not necessarily fail on an instance that does not meet our assumptions. However, we no longer have a guarantee on its running time. Random instances fail to meet the assumption with a small probability, but we empirically observed that the algorithm solves them in reasonable time as well.

\subsection{Counting Linearly Independent Equations}

Obtaining guarantees on the number of linearly independent equations in $\mathcal{S}$ is the most important and the most delicate part of the attack. Since dim ker $\mathcal{S}$ is a function of the instance, it makes sense to consider the random variable giving $\operatorname{dim} \operatorname{ker} \mathcal{S}$ assuming the instance was randomly chosen. Fig. 1 above shows its (experimentally observed) distribution for various sizes of the base field. We immediately see that in odd characteristic, $\operatorname{dim} \operatorname{ker} \mathcal{S}$ is often $n$, while in characteristic two it is often $2 n$. In the sequel we provide mathematical arguments to back this observation up. We will focus on the (harder) case of fields of characteristic two, since this is the more cryptographically relevant case.

Our results are expressed in terms of the similarity invariants $P_{1}, \ldots, P_{s}$ of a matrix $M$. Their product is the characteristic polynomial of $M, P_{s}$ is the minimal polynomial of $M$, and $P_{i}$ divides $P_{i+1}$. The main technical result needed to understand the rank of $\mathcal{S}$ is the following theorem.

Theorem 1. Let $A_{1}, A_{2}, B_{1}, B_{2}$ be four given matrices of size $n \times n$ with coefficients in $\mathbb{F}_{q}$. Let us consider the set of all pairs $(X, Y)$ of $n \times n$ matrices satisfying the following linear equations: 


$$
\mathcal{S}: \quad\left\{\begin{array}{l}
B_{1}=X \cdot A_{1} \cdot Y \\
B_{2}=X \cdot A_{2} \cdot Y
\end{array}\right.
$$

Let us assume that $\mathcal{S}$ admits at least one solution $\left(X_{0}, Y_{0}\right)$ with both $X_{0}$ and $Y_{0}$ invertible, and that $A_{1}$ is also invertible.

i) There is a vector-space isomorphism between the kernel of $\mathcal{S}$ and the commutant of $\mathcal{C}=A_{2} \cdot A_{1}^{-1}$.

ii) $n \leq \operatorname{dim} \operatorname{ker} \mathcal{S}$.

iii) Let $P_{1}, \ldots, P_{s}$ be the similarity invariants of $\mathcal{C}$. Then:

$$
\operatorname{dim} \operatorname{ker} \mathcal{S}=\sum_{j=1}^{s}(2 s-2 j+1) \cdot \operatorname{deg} P_{j}
$$

Proof. Because a solution of $\mathcal{S}$ exists, then $B_{1}$ is invertible. Thanks to this, we can write:

$$
\mathcal{S}:\left\{\begin{aligned}
Y & =A_{1}^{-1} \cdot X^{-1} \cdot B_{1} \\
B_{2} \cdot B_{1}^{-1} \cdot X & =X \cdot A_{2} \cdot A_{1}^{-1}
\end{aligned}\right.
$$

Using the particular solution $X_{0}$ then gives:

$$
\mathcal{S}:\left\{\begin{aligned}
Y & =A_{1}^{-1} \cdot X^{-1} \cdot B_{1} \\
\mathcal{C} \cdot\left(X_{0}^{-1} \cdot X\right) & =\left(X_{0}^{-1} \cdot X\right) \cdot \mathcal{C}
\end{aligned}\right.
$$

From there, it is not difficult to see that the kernel of $\mathcal{S}$ is in one-to-one correspondance with the commutant of $\mathcal{C}$, the isomorphism being $(X, Y) \mapsto X_{0}^{-1} \cdot X$. The second point of the theorem follows from the well-known fact that $n$ lower-bounds the dimension of the commutant of any endomorphism on a vector space of dimension $n$ (see for instance [7, Fact 2.18.9]). The third point follows from a general result on the dimension of the commutant [20, chapter 6 , exercise 32].

Theorem 1 directly applies to our study of the rank of $\mathcal{S}$ with $A_{i}=\mathcal{P}\left(\mathbf{a}_{i}\right)$ and $B_{i}=$ $\mathcal{P}\left(\mathbf{b}_{i}\right)$. However, it holds only if $\mathcal{P}\left(\mathbf{a}_{1}\right)$ or $\mathcal{P}\left(\mathbf{a}_{2}\right)$ is invertible (we may swap them if we wish, or even take a linear combination). Note that since $\mathcal{P}\left(\mathbf{a}_{1}\right)$ is a random skew-symmetric matrix, it cannot be invertible if $n$ is odd, and the analysis is more complicated in that case. This is why we focus on the case where $n$ is even, and where one of the two quadratic forms is non-degenerate. The following lemma gives us the probability that $\mathcal{P}\left(\mathbf{a}_{i}\right)$ (or $\mathcal{P}\left(\mathbf{b}_{i}\right)$ ) is invertible.

Lemma 2 ([32], theorem 3). Let $N_{0}(n, r)$ denote the number of symmetric matrices of size $n \times n$ over $\mathbb{F}_{q}$ with zeros on the diagonal and of rank $r$.

$$
\begin{aligned}
N_{0}(n, 2 s) & =\prod_{i=1}^{s} \frac{q^{2 i-2}}{q^{2 i}-1} \cdot \prod_{i=1}^{2 s-1}\left(q^{n-i}-1\right) \\
N_{0}(n, 2 s+1) & =0
\end{aligned}
$$


If $n$ is even, the probability that $\mathcal{P}\left(\mathbf{a}_{1}\right)$ is invertible if $q=2$ is about 0.419 (this probability increases exponentially with $q)$. The probability that either $\mathcal{P}\left(\mathbf{a}_{1}\right)$ or $\mathcal{P}\left(\mathbf{a}_{2}\right)$ is invertible is then about 0.662 when $q=2$.

Theorem 1 is then applicable in more than half of the cases when $q=2$ (and we expect this proportion to grow very quickly with $q$ ). When it is applicable, what guarantee does it exactly offer? We would need to know something about the similarity invariants of $\mathcal{C}$. An easy case would be when the minimal and characteristic polynomials are the same (then there is only one invariant factor, and it is precisely the characteristic polynomial). Then Theorem[1tells us that the dimension of $\operatorname{ker} \mathcal{S}$ is $n$. For random matrices, the probability of this event is given by the following lemma.

Lemma 3 ([21], theorem 1). Let $c(n, q)$ be the proportion of cyclic $n \times n$ matrices (i.e., matrices for which the minimal polynomial is of degree $n$ ). We have:

$$
\frac{1}{q^{2}(q+1)}<1-c(n, q)<\frac{1}{\left(q^{2}-1\right)(q-1)}
$$

And asymptotically, we have:

$$
\lim _{n \rightarrow \infty} c(n, q)=\frac{q^{5}-1}{q^{2}(q-1)\left(q^{2}-1\right)} \cdot \prod_{i=1}^{\infty}\left(1-\frac{1}{q^{i}}\right)
$$

For random matrices over $\mathbb{F}_{2}$, and for $n$ big enough, the proportion of cyclic matrices approaches 0.746 . Unfortunately, $\mathcal{C}$ is hardly a random matrix. In odd characteristic it is the product of two symmetric matrices, while in characteristic two it is the product of two symmetric matrices with null diagonal (and these are in fact skew-symmetric matrices). The product of two skew-symmetric matrices is very far from being random, and it is in fact never cyclic, as the following result shows.

Theorem $2([\mathbf{6} \mid)$. Let $M$ be a non-singular matrix of even dimension. Then the two following conditions are equivalent:

i) $M$ can be written as the product of two skew-symmetric matrices .

ii) $M$ has an even number of similarity invariants $P_{1}, \ldots P_{2 \ell}$, and $P_{2 i+1}=P_{2 i+2}$.

Corollary 1. In characteristic two, if $n$ is even and $\mathcal{C}$ is invertible, then $\operatorname{ker} \mathcal{S}$ has dimension at least $2 n$.

Proof. By theorem $2, \mathcal{C}$ has at least two invariants, both equal to the minimal polynomial of $\mathcal{C}$ (which thus happens to be of degree $n / 2$ ). Then theorem 1 point $i$ iii) shows that ker $\mathcal{S}$ has dimension $2 n$. If $\mathcal{C}$ has more invariants, ker $\mathcal{S}$ can only be of higher dimension.

Corollary 1 shows that with a constant probability (when the two quadratic forms are non-degenerate) $\operatorname{dim} \operatorname{ker} \mathcal{S}$ is greater than $2 n$, which sounds like bad news. When $\mathcal{C}$ is not invertible, theorem 2 no longer holds (there are counter-examples), but what does apparently still hold is the fact that the minimal polynomial of $\mathcal{C}$ has degree at most $n / 2$, and this would be sufficient to show that in all cases $\operatorname{dim} \operatorname{ker} \mathcal{S} \geq 2 n$, in accordance with Fig.11. 
What we would in fact need to know is the probability that $\operatorname{ker} \mathcal{S}$ is exactly of dimension $2 n$. Theorem 1 still connects this dimension to the similarity invariants of $\mathcal{C}$, even though $\mathcal{C}$ is not a uniformly random matrix. It seems plausible that $\mathcal{C}$ is unlikely to have a very high number of similarity invariants, and that the most common situation is that it has only two invariants (twice the minimal polynomial). We could not compute explicitly this probability, and we could not find ways to obtain it in the available literature. We measured it experimentally and found 0.746 (after $10^{5}$ trials) when $q=2$. This is strikingly close to the result brought by lemma 3 in the random case. Under the conjecture that $\mathcal{C}$ has two invariant factors with this probability, then theorem 1 tells us that in about $75 \%$ of the cases, $\operatorname{dim} \operatorname{ker} \mathcal{S}=2 n$. The empirical probability seems to be even higher, as shown by Fig. 1 .

\subsection{Solving Very Overdefined Quadratic Systems}

The solution of the IP1S instance (1) is systematically the solution of a system $\mathcal{S}_{\text {quad }}$ of $n^{2}$ quadratic equations. In the previous section, we argued that we can reduce this system to $n^{2}$ equations in $2 n$ unknowns with high probability, and (much) more unknowns with negligible probability. The system is so overdefined that it can almost be resolved by linearization. Indeed, it has $N^{2} / 4$ equations in $N$ unknowns. In practice, computing a Gröbner basis of the ideal generated by $\mathcal{S}_{\text {quad }}$ terminates very quickly, and allows to recover the actual solutions of the problem.

This last fact can be theoretically justified. It is well-known that Gröbner basis algorithms [15,16] are more efficient on overdefined systems. The complexity of most algorithms strongly depend on a parameter of the ideal called the degree of regularity. Indeed, the cost of computing a Gröbner basis is polynomial in the degree of regularity $D_{\text {reg }}$ of the system when the ideal has dimension zero, i.e., when the number of solutions is finite. The computation of a Gröbner basis essentially amounts to solve a system of $M$ sparse linear equations in $M$ variables, where $M$ is the number of monomials of degree $D_{\text {reg }}$ in $N$ variables. The complexity of this process is roughly $\mathcal{O}\left(N^{\omega \cdot D_{\text {reg }}}\right)$, with $2<\omega \leq 3$ the linear algebra constant, and $N$ the number of variables of ideal considered (in our case, $N=2 n$ ).

The behavior of the degree of regularity is well understood for "random" systems of equations [345] (i.e., regular or semi-regular systems). It is conjectured that the proportion of semi-regular systems on $N$ variables goes to 1 when $N$ goes to $+\infty$. Therefore, we can assume that for large $N$ a random system is almost surely semi-regular. This is to some extent a worst-case assumption, as it usually means that our system is not easier to solve than the others. The coefficients of the Hilbert series associated with the ideal generated by a semi-regular sequence of $m$ equations in $N$ variables coincide with those of the series expansion of the function $f(z)=\left(1-z^{2}\right)^{m} /(1-z)^{N}$, up to the degree of regularity. The degree of regularity is the smallest degree $d$ such that the coefficient of degree $d$ in the series expansion of $f(z)$ is not strictly positive. This property enables an explicit computation of the degree of regularity for given values of $m$ and $N$.

Furthermore, the available literature readily provide asymptotic estimates of the degree of regularity for semi-generic ideals of $N+k$ or $\alpha \cdot N$ equations in $N$ variables, but unfortunately not for the case of $\alpha \cdot N^{2}$ in $N$ variables, which is the situation we 
Table 3. Degree of regularity of random with the same parameters as those occuring in our attack

\begin{tabular}{|c||r|r|r|r|r|r|r|r|r|r|c|}
\hline$n$ & 2 & 3 & 4 & 5 & 6 & 7 & 8 & $\ldots$ & 16 & $\ldots$ & 32 \\
\hline$N$ & 4 & 6 & 8 & 10 & 12 & 14 & 16 & $\ldots$ & 32 & $\ldots$ & 64 \\
\hline$m$ & 4 & 9 & 16 & 25 & 36 & 49 & 64 & $\ldots$ & 256 & $\ldots$ & 1024 \\
\hline$D_{\text {reg }}$ & 5 & 4 & 3 & 3 & 3 & 3 & 3 & $\ldots$ & 3 & $\ldots$ & 3 \\
\hline
\end{tabular}

are facing here. We thus tabulated in table. 3 the degree of regularity for semi-regular systems of equations having the same number of equations and unknowns as those occurring in our attack. From this table, we conclude that for any reasonable value of the parameters, the degree of regularity will be 3, and thus computing a Gröbner basis of $\mathcal{S}_{\text {quad }}$ should have complexity at most $\mathcal{O}\left(n^{9}\right)$. In practice, the maximal degree reached by the $\mathrm{F}_{4}$ algorithm on our equations is two, which is even better.

\subsection{Implementation}

We demonstrated that the algorithm described in this section terminates in time $\mathcal{O}\left(n^{6}\right)$ on a constant fraction of the instances. This reasoning is backed up by empirical evidence: we implemented the algorithm using the computer algebra system MAGMA [9]. Solving the equations of $\mathcal{S}_{\text {quad }}$ is achieved by first computing a Gröbner basis of these equations for the Graded-Reverse Lexicographic order using the $\mathrm{F}_{4}$ algorithm [15], and then converting it to the Lexicographic order using the FGLM algorithm [14]. This implementation breaks the random instances of IP1S in very practical time. For instance, Challenges $\mathrm{A}$ and $\mathrm{C}$ are solved in a few seconds. Random instances with $n=24, u=2$ require about a minute. Challenge $\mathrm{E}$ takes about 10 minutes, but the dominating part in the execution of the algorithm is in fact the symbolic manipulation of polynomials required to write down the equations of $\mathcal{S}_{\text {quad }}$. Actually solving the resulting quadratic equations turns out to be easier than generating them. We never generated a random instance that we could not solve with our technique, for any choice of the parameters.

There are only public parameter sets, and no public challenges to break, so we unfortunately cannot provide the solution of an open challenge to prove that our algorithm works. However, the source code of our implementation is available on the webpage of the first author.

\section{Cryptanalysis of Cubic IP1S}

In this section, we focus on the case where $\mathbf{a}$ and $\mathbf{b}$ are composed of a single cubic polynomial. We assume that $\mathbf{a}$ and $\mathbf{b}$ are given explicitly, i.e.:

$$
\mathbf{a}=\sum_{i=1}^{n} \sum_{j=i}^{n} \sum_{k=j}^{n} A_{i, j, k} \cdot x_{i} x_{j} x_{k}, \quad \mathbf{b}=\sum_{i=1}^{n} \sum_{j=i}^{n} \sum_{k=j}^{n} B_{i, j, k} \cdot x_{i} x_{j} x_{k} .
$$

As already explained, we can restrict our attention to the homogenous case. The techniques developed previously for the quadratic case cannot directly applied in this 
setting. Indeed, the differential is no longer a bilinear mapping, and then there is no obvious linear equations between the coefficients of a solution and those of its inverse. However, we can combine the use of the differential together with the Gröbner basis approach proposed in [17]. We denote by $S_{0}=\left\{s_{i, j}^{0}\right\}_{1 \leq i, j \leq n}$ a particular solution of IP1S between $\mathbf{a}$ and $\mathbf{b}$, i.e., it holds that $\mathbf{b}=\mathbf{a} \circ S_{0}$. For all vectors $\mathbf{x}, \mathbf{y} \in\left(\mathbb{F}_{q}\right)^{n}$, we have:

$$
\operatorname{Da}\left(S_{0} \cdot \mathbf{x}, \mathbf{y}\right)=\operatorname{Db}\left(\mathbf{x}, S_{0}^{-1} \cdot \mathbf{y}\right)
$$

$\mathbf{a}$ and $\mathbf{b}$ being of total degree 3 , the coefficients of $S_{0}$ and $S_{0}^{-1}$ appear with degree two in the expression of $\mathrm{Da}$ and $\mathrm{Db}$ above. Let $R$ be the ring $\mathbb{K}\left[s_{1,1}, \ldots, s_{n, n}, u_{1,1}, \ldots, u_{n, n}\right]$. We consider the algebra $\mathcal{A}^{s}$ of all $n \times n$ matrices over $R$. Let $S=\left\{s_{i, j}\right\}$ and $U=\left\{u_{i, j}\right\}$ in $\mathcal{A}^{s}$ be symbolic matrices. We denote by $\mathcal{I}_{\mathbf{a}, \mathbf{b}}$ the ideal generated by all the coefficients in $R$ of the equations:
$\operatorname{Da}(S \cdot \mathbf{x}, \mathbf{y})-\operatorname{Db}(\mathbf{x}, U \cdot \mathbf{y})=0$
$U \cdot S-1_{n}=0_{n}$,
$S \cdot U-1_{n}=0_{n}$.

It is easy to see that $U=S_{0}^{-1}$ and $S=S_{0}$ is a particular solution of this system, and also a solution of IP1S between $\mathbf{b}$ and $\mathbf{a}$. Our goal is to provide an upper bound on the maximum degree reached during a Gröbner basis computation of $\mathcal{I}_{\mathbf{a}, \mathbf{b}}$.

We prove here that $D_{\text {reg }}=2$ for $\mathcal{I}_{\mathbf{a}, \mathbf{b}}$ under the hypothesis that we know one row of a particular solution $S_{0}$, i.e., we assume then that we know the following ideal $\mathcal{J}=$ $\left\langle s_{1, j}-s_{1, j}^{(0)} \mid j=1, \ldots, n\right\rangle$.

Theorem 3. The degree of regularity of $\mathcal{I}_{\mathbf{a}, \mathbf{b}}+\mathcal{J}$ is 2 . Therefore, computing a Gröbner basis of this ideal takes time $\mathcal{O}\left(n^{6}\right)$.

Proof. We use the fact that the degree of regularity of an ideal is generically left invariant by any linear change of the variables or generators [29]. In particular, we consider the ideal $\mathcal{I}_{\mathbf{a}, \mathbf{b}}^{\prime}$ generated by all the coefficients in $\mathbb{K}\left[x_{1}, \ldots, x_{n}, y_{1}, \ldots, y_{n}\right]$ of the equations:

$\operatorname{Da}\left(S_{0}\left(S+I_{n}\right) \mathbf{x}, \mathbf{y}\right)-\operatorname{Db}\left(\mathbf{x},\left(U+I_{n}\right) S_{0}^{-1} \mathbf{y}\right)=0, \quad U \cdot S=0_{n}, \quad S \cdot U=0_{n}$.

It is clear that $\mathcal{I}_{\mathbf{a}, \mathbf{b}}^{\prime}$ is obtained from $\mathcal{I}_{\mathbf{a}, \mathbf{b}}$ by replacing $S$ (resp. $U$ ) by $S_{0}\left(I_{n}+S\right)$ (resp. $\left.\left(U+I_{n}\right) S_{0}^{-1}\right)$. Thus, the degree of regularity of $\mathcal{I}_{\mathbf{a}, \mathbf{b}}^{\prime}$ and $\mathcal{I}_{\mathbf{a}, \mathbf{b}}$ are equal. Using the same transformation, the ideal $\mathcal{J}$ becomes

$$
\mathcal{J}^{\prime}=\left\langle s_{1, j} \mid j=1, \ldots, n\right\rangle \text {. }
$$

We now estimate the degree of regularity of the ideal $\mathcal{I}_{\mathbf{a}, \mathbf{b}}^{\prime}+\mathcal{J}^{\prime}$. For a reason which will become clear in the sequel, it is more convenient to work with $\mathcal{I}_{\mathbf{a}, \mathbf{b}}^{\prime}+\mathcal{J}^{\prime}$. In what follows, $F$ will denote the generators of $\mathcal{I}_{\mathbf{a}, \mathbf{b}}^{\prime}+\mathcal{J}^{\prime}$. We will show that many new linear equations appear when considering equations of degree 2 . To formalize this, we introduce some definitions related to the $\mathrm{F}_{4}$ algorithm [16]. In particular, we will denote by $I_{d, k}$ the linear space generated during the $k$-th step of $\mathrm{F}_{4}$ when considering polynomials of degree $d$. 
Definition 1. We have the following recursive definition of $I_{d, k}$ :

$$
\begin{aligned}
I_{d, 0}(F)= & \operatorname{Vect}_{\mathbb{K}}(F) \\
I_{d, 1}(F)= & \operatorname{Vect}_{\mathbb{K}}\left(s_{i, j} f \mid 1 \leqslant i, j \leqslant n \text { and } f \in I_{d, 0}(F)\right) \\
& +\operatorname{Vect}_{\mathbb{K}}\left(u_{i, j} f \mid 1 \leqslant i, j \leqslant n \text { and } f \in I_{d, 0}(F)\right) \\
I_{d, k}(F)= & \operatorname{Vect}_{\mathbb{K}}\left(s_{i, j} f \mid 1 \leqslant i, j \leqslant n \text { and } f \in I_{d, k-1}(F) \text { and } \operatorname{deg}(f) \leq d-1\right) \\
& +\operatorname{Vect}_{\mathbb{K}}\left(u_{i, j} f \mid 1 \leqslant i, j \leqslant n \text { and } f \in I_{d, k-1}(F) \text { and } \operatorname{deg}(f) \leq d-1\right) .
\end{aligned}
$$

Roughly speaking, the index $k$ is the number of steps in the $F_{4} / F_{5}$ [16] algorithm to compute an element $f \in I_{d, k}(F)$. We show that $I_{2,1}(F)$ contains exactly $n^{2}+2 n$ linear equations. This means that we have already many linear equations generated during the first step of a Gröbner basis computation of $F$.

Lemma 4. $I_{2,1}(F)$ contains the following linear equations:

$$
\left\{u_{1, j} \mid j=1, \ldots, n\right\} .
$$

Proof. From the first row of the following zero matrix $S \cdot U$ we obtain the following equations:

$$
\left\{\begin{array}{c}
s_{1,1} u_{1,1}+s_{1,2} u_{2,1}+s_{1,3} u_{3,1}+\cdots+s_{1, n} u_{n, 1}=0 \\
s_{1,1} u_{1,2}+s_{1,2} u_{2,2}+s_{1,3} u_{3,2}+\cdots+s_{1, n} u_{n, 2}=0 \\
s_{1,1} u_{1,3}+s_{1,2} u_{2,3}+s_{1,3} u_{3,3}+\cdots+s_{1, n} u_{n, 3}=0 \\
\ldots \\
s_{1,1} u_{1, n}+s_{1,2} u_{2, n}+s_{1,3} u_{3, n}+\cdots+s_{1, n} u_{n, n}=0
\end{array}\right.
$$

Using the equations $s_{1, j}=0$ from the ideal $\mathcal{J}^{\prime}$, we obtain then $u_{1,1}=0, u_{1,2}=$ $0, \ldots, u_{1, n}=0$.

We can also predict the existence of other linear equations in $I_{2,1}(F)$.

Lemma 5. For all $(i, j) \in\{1, \ldots, n\}^{2}$ the coefficient of $y_{1} y_{i} x_{j}$ in $\operatorname{Da}\left(S_{0}(S+\right.$ $\left.\left.I_{n}\right) \mathbf{x}, \mathbf{y}\right)-\mathrm{Db}\left(\mathbf{x},\left(U+I_{n}\right) S_{0}^{-1} \mathbf{y}\right)$ is a non zerd 4 linear equation modulo the equations of the ideal $\mathcal{J}^{\prime}$ and (4). Among these equations, there are $n$ which depend only of the variables $\left\{s_{k, \ell} \mid 1 \leq k, \ell \leq n\right\}$.

Proof. We consider the coefficient of the monomial $m=y_{1} y_{i} x_{j}$ in the expression

$$
\Delta=\Delta_{a}-\Delta_{b}=\operatorname{Da}\left(S_{0}\left(S+I_{n}\right) \mathbf{x}, \mathbf{y}\right)-\operatorname{Db}\left(\mathbf{x},\left(U+I_{n}\right) S_{0}^{-1} \mathbf{y}\right) .
$$

Since the monomial $m$ is linear in $x_{j}$ it is clear that the corresponding coefficient in $\Delta_{a}=\mathrm{Da}\left(S_{0}\left(S+I_{n}\right) \mathbf{x}, \mathbf{y}\right)$ is also linear in the variables $s_{i, j}$; moreover this coefficient is non zero. We have now to consider the coefficient of $m$ in $\Delta_{b}$. Since $\operatorname{Db}(\mathbf{x}, \mathbf{y})$ is the differential of an homogenous polynomial of degree 3 we can always write:

$$
\operatorname{Db}(\mathbf{x}, \mathbf{y})=\sum_{i=1}^{n} \sum_{j=i}^{n} \ell_{i, j}\left(y_{1}, \ldots, y_{n}\right) x_{i} x_{j}+\sum_{i=1}^{n} q_{i}\left(y_{1}, \ldots, y_{n}\right) x_{i}
$$

\footnotetext{
${ }^{4}$ More precisely, generically non zero.
} 
where $\ell_{i, j}$ (resp. $q_{i}$ ) is a polynomial of degree 1 (resp. 2). Consequently, the coefficient of $m$ in $\mathrm{Db}$ is also the coefficient of $y_{1} y_{i}$ in $q_{j}\left(\left(U+I_{n}\right) S_{0}^{-1} \mathbf{y}\right)$. That is to say, in $q_{j}(\mathbf{y})$ we have now to replace $\mathbf{y}=\left(y_{1}, \ldots, y_{n}\right)$ by $\left(\left(U+I_{n}\right) S_{0}^{-1} \mathbf{y}\right)$. Thus, modulo the equations of the ideal $\mathcal{J}^{\prime}$ and (4), we can write the product $\left(\left(U+I_{n}\right) S_{0}^{-1} \mathbf{y}\right)$ as

$$
\begin{aligned}
& =\left(\begin{array}{c}
y_{1} \\
\vdots \\
\vdots \\
y_{n}
\end{array}\right)\left(\begin{array}{cccc}
1 & 0 & 0 & 0 \\
u_{2,1} & \cdots & \cdots & u_{2, n} \\
\vdots & \cdots & \cdots & \vdots \\
u_{n, 1} & \cdots & \cdots & u_{n, n}
\end{array}\right)\left(\begin{array}{c}
* * * * \\
* * * * \\
* * * * \\
* * * *
\end{array}\right)
\end{aligned}
$$

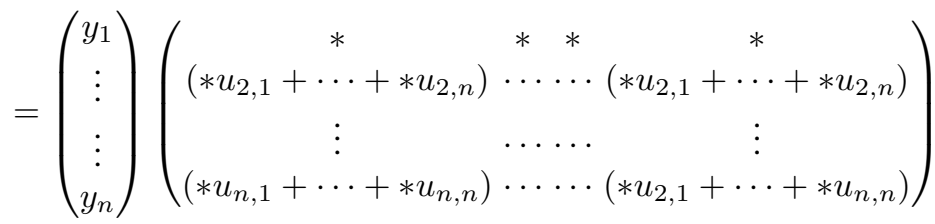

$$
\begin{aligned}
& =\left(\begin{array}{c}
* y_{1}+\left(* u_{2,1}+\cdots+* u_{2, n}\right) y_{2}+\cdots+\left(* u_{n, 1}+\cdots+* u_{n, n}\right) y_{n} \\
* y_{1}+\left(* u_{2,1}+\cdots+* u_{2, n}\right) y_{2}+\cdots+\left(* u_{n, 1}+\cdots+* u_{n, n}\right) y_{n} \\
\vdots \\
* y_{1}+\left(* u_{2,1}+\cdots+* u_{2, n}\right) y_{2}+\cdots+\left(* u_{n, 1}+\cdots+* u_{n, n}\right) y_{n}
\end{array}\right)
\end{aligned}
$$

Hence the coefficient of $y_{1} y_{i}$ in $q_{j}\left(\left(U+I_{n}\right) S_{0}^{-1} \mathbf{y}\right)$ is linear in the variables $u_{k, l}$ when $i \neq 1$ and the coefficient of $y_{1}^{2}$ is a constant.

To summarize:

Lemma 6. $I_{2,1}(F)$ contains exactly $n^{2}+2 n$ linear equations.

Proof. In $I_{2,1}(F)$, we have $n$ linear equations from lemma 5 , $n$ linear equations from the very definition of $\mathcal{J}^{\prime}$, and $n^{2}$ linear equations from lemma 5

As explained before, we obtain $n^{2}+2 n$ linear equations for $I_{2,1}(F)$. However, we have $2 n^{2}$ variables. So, we have to consider $I_{2,2}(F)$, i.e., the equations generated at degree 2 during the second step. Thanks to lemma 6 we can reduce the original system to a quadratic system in $2 n^{2}-\left(2 n+n^{2}\right)=(n-1)^{2}$ variables. W.l.o.g we can assume that we keep only the variable $u_{i, j}$ where $2 \leq i, j \leq n$. Let $F^{\prime}$ be the system obtained from $F$ after substituting the $2 n+n^{2}$ linear equations of lemma 6 , All the monomials in $\mathbb{K}\left[x_{1}, \ldots, x_{n}, y_{1}, \ldots, y_{n}\right]$ of $\operatorname{Da}\left(S_{0}\left(S+I_{n}\right) \mathbf{x}, \mathbf{y}\right)-\operatorname{Db}\left(\mathbf{x},\left(U+I_{n}\right) S_{0}^{-1} \mathbf{y}\right)$ have the following shape:

$$
x_{i} y_{j} y_{k} \text { or } y_{i} x_{j} x_{k} \text { with } 1 \leq i, j, k \leq n .
$$

Hence the number of such monomials is $2 n \frac{n(n+1)}{2}=n^{2}(n+1) \approx n^{3}$, which implies that the number of equations in $F^{\prime}$ is also $n^{3}$.

Thanks to this remark, we will now prove that we can linearize $F^{\prime}$. Let $T\left(F^{\prime}\right)$ be the set of all monomials occurring in $F^{\prime}$. We can assume that $T\left(G^{\prime}\right)=\left[t_{1}<t_{2}<\cdots<\right.$ $\left.t_{N}\right]$. It is important to remark that $t_{1}=u_{2,2}$ up to $t_{(n-1)^{2}}=u_{n, n}$ are in fact variables. Now, let $M$ be the matrix representation of $G^{\prime}$ w.r.t. $T\left(G^{\prime}\right)$. Since we know precisely the shape of the equations from the proof of lemma 5, it is possible to establish that: 
1. most of the equations are very sparse, namely each equation contains about $n^{2}$ non-zero terms.

2. all the variables $t_{1}, \ldots, t_{(n-1)^{2}}$ occur in all the equations.

After a Gaussian elimination of the matrix $M$, we obtain the following shape:

$$
\widetilde{M}=\left[\begin{array}{cccc}
1_{(n-1)^{2}} & 0 & 0 & 0 \\
0 & \times & \ldots & \ldots \\
0 & \times & \ddots & \vdots \\
0 & \times & \ldots & \ddots
\end{array}\right]
$$

Hence, we obtain after a second step of computation in degree 2 the equations $u_{2,2}=$ $\cdots=u_{n, n}=0$. This means that after 2 steps of computation at degree 2 , we obtain $(n-1)^{2}+2 n+n^{2}=2 n^{2}$ linear equations in $2 n^{2}$ unknowns. This explains why the maximum degree reached during the Gröbner basis computation of $\mathcal{I}_{\mathbf{a}, \mathbf{b}}^{\prime}+\mathcal{J}^{\prime}$ is bounded by 2 , and concludes the proof of theorem 3 .

\subsection{Application to the Linear Inhomogeneous Case}

If $c=0$ in equation (1), and if a has a non-trivial homogeneous component of degree one, then looking at the homogeneous component of degree one yields the image of $S$ on one point. We are then in a situation where theorem 3 is applicable, and $S$ can be determined though a Gröbner basis computation which terminates in time $\mathcal{O}\left(n^{6}\right)$.

\subsection{Implementation and Application to the Other Cases}

All the other cases reduce to the linear homogeneous case, as mentioned in section 2 In this setting, the problem is that we do not have enough knowledge on $S$ to make the Gröbner basis computation efficient. A simple idea would be to guess a column of $S$ then compute the Gröbner basis. This approach has complexity $\mathcal{O}\left(n^{6} \cdot q^{n}\right)$ as explained before. It is possible to reduce this complexity by a factor of $q$, by discarding guesses for the column of $S$ that yields different values of $\mathbf{a}$ and $\mathbf{b}$ on the corresponding points.

The biggest proposed cubic IP1S challenge (Challenge $\mathrm{C}$ in fig. 2) has $u=1, n=16$ and $q=2$. Given one relation on $S$, the computation of the Gröbner basis takes 90 seconds on a $2.8 \mathrm{Ghz}$ Xeon computer using the publicly available implementation of $\mathrm{F}_{4}$ in MAGMA. Since this has to be repeated $2^{15}$ times, the whole process takes about one CPU-month (and can be parallelized at will). For challenge D, the Gröbner basis is computed in 0.1 second, and the whole process takes about 2 hours.

\subsection{An Interesting Failure}

We conclude this section with a simple idea that could have lead to an improvement, by efficiently giving a relation on $S$, but which fails in an interesting manner. Let us assume that $\mathbf{a}$ and $\mathbf{b}$ are homogeneous, and that $c=0$ (in that setting, if $c \neq 0$, then $c$ can be retrieved following the observation of [24]). Let us denote by $Z_{\mathbf{a}}$ (resp. $Z_{\mathbf{b}}$ ) the set of zeroes of a (resp. b). Because of lemma 1 , and since $S$ is linear, we have: 


$$
S\left(\sum_{\mathbf{x} \in Z_{\mathbf{a}}} \mathbf{x}\right)=\sum_{\mathbf{y} \in Z_{\mathbf{b}}} \mathbf{y}
$$

This yields a relation on $S$, which is enough to use theorem 3 a and $\mathbf{b}$ may be assumed to have about $q^{n-1}$ zeroes. Finding them requires time $\mathcal{O}\left(q^{n}\right)$. The complexity of the attack could thus be improved to $\mathcal{O}\left(n^{6}+q^{n}\right)$. Surprisingly, this trick fails systematically, and this happen to be consequence of the Chevalley-Warning theorem [1046].

Lemma 7. The sum of the zeroes of a cubic form on 5 variables or more over $\mathbb{F}_{q}$ is always zero.

Proof. Let us consider the elements of $Z_{\mathbf{a}}$ having $\alpha$ as their first coordinate, and let us denote by $n_{\alpha}$ their number. These are in fact the common zeroes of $\left(\mathbf{a}, x_{1}-\alpha\right)$. By the Chevalley-Warning theorem [1046], if a has at least 5 variables, then the characteristic of the field divides $n_{\alpha}$. Therefore, their sum has zero on the first coordinate. Applying this result for all values of $\alpha$ shows that the sum of zeroes of a has a null first coordinate. We then just consider all coordinates successively.

\section{Conclusion}

In this paper, we present algorithms for the IP problem with one secret for two random quadratic equations and one cubic equation. As already explained, there are the most cryptographically relevant instances. Moreover, we explain the complexity, success probability and give sufficient conditions so that the algorithms work. We combine the use of the differential and the computation of Gröbner bases of very overdefined systems. All the proposed IP1S challenges can be broken in practice by the technique we describe, as the following table shows.

\begin{tabular}{|c|c|}
\hline Challenge & Attack time on one core \\
\hline \hline A & 3 seconds \\
\hline B & 1 month \\
\hline C & 0 seconds \\
\hline D & 1 hours \\
\hline E & 3 minutes \\
\hline
\end{tabular}

In view of these results, we conclude that Patarin's IP1S-Based identification scheme is no longer competitive with respect to others combinatorial-based identification schemes [42 43 44445].

Acknowledgement. The first author thanks Mehdi Tibouchi for pointing out the existence of the Chevalley-Warning theorem, and for interesting discussions in general. The work described in this paper has been supported in part by the European Commission through the ICT programme under contract ICT-2007-216676 ECRYPT II. The two last authors were also supported in part by the french ANR under the Computer Algebra and Cryptography (CAC) project ANR-09- JCJCJ-0064-01. 


\section{References}

1. Baena, J., Clough, C., Ding, J.: Square-vinegar signature scheme. In: Buchmann, J., Ding, J. (eds.) PQCrypto 2008. LNCS, vol. 5299, pp. 17-30. Springer, Heidelberg (2008)

2. Bardet, M., Faugère, J.C., Salvy, B., Yang, B.Y.: Asymptotic Behaviour of the Degree of Regularity of Semi-Regular Polynomial Systems. In: MEGA 2005, Eighth International Symposium on Effective Methods in Algebraic Geometry, Porto Conte, Alghero, Sardinia (Italy), May 27-June 1 (2005)

3. Bardet, M.: Étude des systèmes algébriques surdéterminés. Applications aux codes correcteurs et à la cryptographie. PhD thesis, Université de Paris VI (2004)

4. Bardet, M., Faugère, J.-C., Salvy, B.: On the complexity of Gröbner basis computation of semi-regular overdetermined algebraic equations. In: Proc. International Conference on Polynomial System Solving (ICPSS), pp. 71-75 (2004)

5. Bardet, M., Faugère, J.-C., Salvy, B., Yang, B.-Y.: Asymptotic behaviour of the degree of regularity of semi-regular polynomial systems. In: Proc. of MEGA 2005, Eighth International Symposium on Effective Methods in Algebraic Geometry (2005)

6. Bennett, A.A.: Products of skew-symmetric matrices. American M. S. Bull. 25, 455-458 (1919)

7. Bernstein, D.S.: Matrix mathematics. Theory, facts, and formulas, 2nd expanded edn., vol. xxxix, p. 1139. Princeton University Press, Princeton (2009)

8. Billet, O., Gilbert, H.: A traceable block cipher. In: Laih, C.S. (ed.) ASIACRYPT 2003. LNCS, vol. 2894, pp. 331-346. Springer, Heidelberg (2003)

9. Bosma, W., Cannon, J.J., Playoust, C.: The Magma Algebra System I: The User Language. J. Symb. Comput. 24(3/4), 235-265 (1997)

10. Chevalley, C.: Démonstration d'une hypothèse de M. Artin. Abh. Math. Semin. Hamb. Univ. 11, 73-75 (1935)

11. Clough, C., Baena, J., Ding, J., Yang, B.-Y., Chen, M.-s.: Square, a new multivariate encryption scheme. In: Fischlin, M. (ed.) CT-RSA 2009. LNCS, vol. 5473, pp. 252-264. Springer, Heidelberg (2009)

12. Ding, J., Wolf, C., Yang, B.-Y.: $\ell$-invertible cycles for multivariate quadratic public key cryptography. In: Okamoto, T., Wang, X. (eds.) PKC 2007. LNCS, vol. 4450, pp. 266-281. Springer, Heidelberg (2007)

13. dit Vehel, F.L., Perret, L.: Polynomial Equivalence Problems and Applications to Multivariate Cryptosystems. In: Johansson, T., Maitra, S. (eds.) INDOCRYPT 2003. LNCS, vol. 2904, pp. 235-251. Springer, Heidelberg (2003)

14. Faugère, J.-C., Gianni, P., Lazard, D., Mora, T.: Efficient Computation of Zero-Dimensional Gröbner Bases by Change of Ordering. Journal of Symbolic Computation 16(4), 329-344 (1993)

15. Faugère, J.-C.: A new efficient algorithm for computing Gröbner bases (F4). Journal of Pure and Applied Algebra 139(1-3), 61-88 (1999)

16. Faugère, J.-C.: A New Efficient Algorithm for Computing Gröbner Bases Without Reduction to Zero (F5). In: ISSAC 2002: Proceedings of the 2002 International Symposium on Symbolic and Algebraic Computation, pp. 75-83. ACM, New York (2002)

17. Faugère, J.-C., Perret, L.: Polynomial Equivalence Problems: Algorithmic and Theoretical Aspects. In: Vaudenay, S. (ed.) EUROCRYPT 2006. LNCS, vol. 4004, pp. 30-47. Springer, Heidelberg (2006)

18. Fiat, A., Shamir, A.: How to prove yourself: Practical solutions to identification and signature problems. In: Odlyzko, A.M. (ed.) CRYPTO 1986. LNCS, vol. 263, pp. 186-194. Springer, Heidelberg (1987)

19. Fortin, S.: The graph isomorphism problem. Technical report, University of Alberta (1996) 
20. Fuhrmann, P.A.: A polynomial approach to linear algebra. Springer, New York (1996)

21. Fulman, J.: Random matrix theory over finite fields. Bull. Amer. Math. Soc. (N.S) 39, 51-85

22. Garey, M.R., Johnson, D.S.: Computers and Intractability, A Guide to the Theory of NP Completeness. Freeman, New York (1979)

23. Geiselmann, W., Meier, W., Steinwandt, R.: An Attack on the Isomorphisms of Polynomials Problem with One Secret. Int. J. Inf. Sec. 2(1), 59-64 (2003)

24. Geiselmann, W., Steinwandt, R., Beth, T.: Attacking the Affine Parts of SFLASH. In: Honary, B. (ed.) Cryptography and Coding 2001. LNCS, vol. 2260, pp. 355-359. Springer, Heidelberg (2001)

25. Goldreich, O., Micali, S., Wigderson, A.: Proofs that yield nothing but their validity and a methodology of cryptographic protocol design (extended abstract). In: FOCS, pp. 174-187. IEEE, Los Alamitos (1986)

26. Goldwasser, S., Micali, S., Rackoff, C.: The knowledge complexity of interactive proofsystems (extended abstract). In: STOC, pp. 291-304. ACM, New York (1985)

27. Kipnis, A., Patarin, J., Goubin, L.: Unbalanced Oil and Vinegar Signature Schemes. In: Stern, J. (ed.) EUROCRYPT 1999. LNCS, vol. 1592, pp. 206-222. Springer, Heidelberg (1999)

28. Koblitz, N.: Algebraic Aspects of Cryptography. Algorithms and Computation in Mathematics, vol. 3. Springer, Heidelberg (1998)

29. Lazard, D.: Gröbner-bases, gaussian elimination and resolution of systems of algebraic equations. In: van Hulzen, J.A. (ed.) ISSAC 1983 and EUROCAL 1983. LNCS, vol. 162, pp. 146-156. Springer, Heidelberg (1983)

30. Lidl, R., Niederreiter, H.: Finite fields. Cambridge University Press, New York (1997)

31. Lyubashevsky, V.: Lattice-based identification schemes secure under active attacks. In: Cramer, R. (ed.) PKC 2008. LNCS, vol. 4939, pp. 162-179. Springer, Heidelberg (2008)

32. MacWilliams, J.: Orthogonal matrices over finite fields. The American Mathematical Monthly 76(2), 152-164 (1969)

33. Matsumoto, T., Imai, H.: Public quadratic polynomial-tuples for efficient signatureverification and message-encryption. In: Günther, C.G. (ed.) EUROCRYPT 1988. LNCS, vol. 330, pp. 419-453. Springer, Heidelberg (1988)

34. Naccache, D. (ed.): CT-RSA 2001. LNCS, vol. 2020. Springer, Heidelberg (2001)

35. Patarin, J.: Hidden fields equations (hfe) and isomorphisms of polynomials (ip): Two new families of asymmetric algorithms. In: Maurer, U.M. (ed.) EUROCRYPT 1996. LNCS, vol. 1070, pp. 33-48. Springer, Heidelberg (1996)

36. Patarin, J.: Hidden Fields Equations (HFE) and Isomorphisms of Polynomials (IP): Two New Families of Asymmetric Algorithms. In: Maurer, U.M. (ed.) EUROCRYPT 1996. LNCS, vol. 1070, pp. 33-48. Springer, Heidelberg (1996), http: / / www . minrank.org/hfe.pdf

37. Patarin, J.: The Oil and Vinegar signature scheme. Presented at the Dagstuhl Workshop on Cryptography (1997)

38. Patarin, J., Courtois, N., Goubin, L.: Flash, a fast multivariate signature algorithm. In: [34], pp. 298-307

39. Patarin, J., Courtois, N., Goubin, L.: QUARTZ, 128-Bit Long Digital Signatures. In: [34], pp. 282-297

40. Patarin, J., Goubin, L., Courtois, N.: Improved Algorithms for Isomorphisms of Polynomials. In: Nyberg, K. (ed.) EUROCRYPT 1998. LNCS, vol. 1403, pp. 184-200. Springer, Heidelberg (1998)

41. Perret, L.: A Fast Cryptanalysis of the Isomorphism of Polynomials with One Secret Problem. In: Cramer, R. (ed.) EUROCRYPT 2005. LNCS, vol. 3494, pp. 354-370. Springer, Heidelberg (2005) 
42. Pointcheval, D.: A new identification scheme based on the perceptrons problem. In: Guillou, L.C., Quisquater, J.-J. (eds.) EUROCRYPT 1995. LNCS, vol. 921, pp. 319-328. Springer, Heidelberg (1995)

43. Shamir, A.: An efficient identification scheme based on permuted kernels (extended abstract). In: Brassard, G. (ed.) CRYPTO 1989. LNCS, vol. 435, pp. 606-609. Springer, Heidelberg (1990)

44. Stern, J.: A new identification scheme based on syndrome decoding. In: Stinson, D.R. (ed.) CRYPTO 1993. LNCS, vol. 773, pp. 13-21. Springer, Heidelberg (1994)

45. Stern, J.: Designing identification schemes with keys of short size. In: Desmedt, Y. (ed.) CRYPTO 1994. LNCS, vol. 839, pp. 164-173. Springer, Heidelberg (1994)

46. Warning, E.: Bemerkung zur vorstehenden Arbeit von Herrn Chevalley.. Abh. Math. Semin. Hamb. Univ. 11, 76-83 (1935)

47. Wolf, C., Preneel, B.: Taxonomy of Public Key Schemes Based on the Problem of Multivariate Quadratic Equations. Cryptology ePrint Archive, Report 2005/077 (2005) 